\title{
ON AN ANALYTICAL DECOMPOSITION OF THE POISSON LAW $\left({ }^{1}\right)$
}

\author{
BY \\ R. G. LAHA
}

The well-known theorem of Cramér [1] on the decomposition of the normal law has been extended by Linnik and Zinger [7] in a purely analytical direction. This generalization is as follows:

Let $f_{1}(t), f_{2}(t), \ldots, f_{N}(t)$ be some characteristic functions and let $\alpha_{1}, \alpha_{2}, \ldots, \alpha_{N}$ be some positive numbers. Let the functions $f_{n}(t)(n=1,2, \ldots, N)$ satisfy the equation $\left({ }^{2}\right)$

$$
\prod_{n=1}^{N}\left\{f_{n}(t)\right\}^{\alpha_{n}}=\exp \left\{i \mu t-\frac{1}{2} \sigma^{2} t^{2}\right\}
$$

for all real $t$ in a certain neighborhood of the point $t=0$, where $\sigma^{2}>0$ and $\mu$ are real numbers. Then each factor $f_{n}(t)$ is the characteristic function of a normal distribution. A similar analytical extension of the theorem of Raikov [10] on the decomposition of the Poisson law has been proven by Dugué [2]. The author [5] has given an alternative proof of this theorem of Dugué. Similar generalizations concerning the decomposition of analytic characteristic functions (characteristic functions which are regular in a horizontal strip containing the real axis) were obtained by Dugué [2], Linnik [6] and the author [4].

Recently Mamay [9] has obtained an interesting generalization of this decomposition theorem of analytic characteristic functions for the case of a denumerable number of factors. This theorem may be stated as follows:

Let $\left\{f_{n}(t)\right\}, n=1,2, \ldots$, be a sequence of characteristic functions and let $\left\{\alpha_{n}\right\}$ be a sequence of positive numbers such that $\alpha_{n} \geqq \alpha_{0}>0$. Let $f(z)$ be an analytic function of the complex variable $z(z=t+i v, t$ and $v$ both real) which is regular in the strip $|\operatorname{Im} z|<M$ and has no zeros inside this strip. Let the functions $f_{n}(t)$ satisfy the equation

$$
\prod_{n=1}^{\infty}\left\{f_{n}(t)\right\}^{\alpha_{n}}=f(t)
$$

for all real $t$ in a certain neighborhood $|t|<\delta(\delta>0)$ of the point $t=0$. Then each

Received by the editors June 12, 1963 and, in revised form, August 19, 1968. 9968.

(1) This work was supported by the National Science Foundation through grant NSF-G-

$\left(^{2}\right)$ For the definition of $\left\{f_{n}(t)\right\}^{\alpha_{n}}=\exp \left\{\alpha_{n} \ln f_{n}(t)\right\}$ we consider that particular branch of the logarithm for which $\ln f_{n}(0)=0$. 
factor $f_{n}(z)$ as a function of the complex variable $z$ is also regular at least in the same strip and further the above equation holds for all complex $z$ in the strip $|\operatorname{Im} z|<M$.

As a direct consequence of this theorem she has also derived an extension of the theorem of Linnik and Zinger [7] for the case of a denumerable number of factors. In the present paper we shall deduce a similar result on the analytical decomposition of the Poisson law for the case of a denumerable number of factors. We formulate this theorem as follows:

THEOREM. Let $\left\{f_{n}(t)\right\}$ be a sequence of characteristic functions and let $\left\{\alpha_{n}\right\}$ be a sequence of positive numbers such that $\alpha_{n} \geqq \alpha_{0}>0$, for $n=1,2, \ldots$ Suppose that the functions $f_{n}(t)$ satisfy the equation

$$
\prod_{n=1}^{\infty}\left\{f_{n}(t)\right\}^{\alpha_{n}}=\exp \{\lambda(\exp \{i t\}-1)\} \quad(\lambda>0)
$$

for all real $t$ in a certain neighborhood $|t|<\delta(\delta>0)$ of the point $t=0$. Then each factor $f_{n}(t)$ has the form

$$
f_{n}(t)=\exp \left\{i \mu_{n} t+\lambda n(\exp \{i t\}-1)\right\}, \quad n=1,2, \ldots,
$$

where $\lambda_{n} \geqq 0$ and $\mu_{n}$ are real numbers.

Before proceeding to the proof of the theorem, we first note that we can assume each $\alpha_{n} \leqq 1(n=1,2, \ldots)$ without any loss of generality, since in case any $\alpha_{n}>1$, there always exists a positive integer $N_{n}$ such that $\alpha_{n} \leqq N_{n}$ and hence we have

$$
\left\{f_{n}(t)\right\}^{\alpha_{n}}=\left\{\left[f_{n}(t)\right]^{N_{n}}\right\}^{\alpha_{n} / N_{n}}=\left\{\psi_{n}(t)\right\}^{\alpha_{n} / N_{n}},
$$

where $\psi_{n}(t)=\left\{f_{n}(t)\right\}^{N_{n}}$ and $\alpha_{n} / N_{n} \leqq 1$. The functions $\psi_{n}(t)$ now satisfy the equation (1) and we deduce from the theorem that each $\psi_{n}(t)$ is of the form (2). Then it follows at once from the theorem of Raikov [10] that each factor $f_{n}(t)$ has also the form (2).

We shall prove this theorem in a series of lemmas.

LEMMA 1. Under the condition of the theorem each factor $f_{n}(t)$ is the characteristic function of a lattice distribution with a span equal to unity.

Proof. It follows immediately from the theorem of Mamay stated above that each factor $f_{n}(z)$ as a function of the complex variable $z(z=t+i v, t$ and $v$ both real) is an entire characteristic function without zeros and further the equation

$$
\prod_{n=1}^{\infty}\left\{f_{n}(z)\right\}^{\alpha_{n}}=\exp \{\lambda(\exp \{i z\}-1)\}
$$

holds for all complex $z$. We put $z=2 \pi$ in equation (3) and thus obtain

$$
\prod_{n=1}^{\infty}\left|f_{n}(2 \pi)\right|^{\alpha_{n}}=1
$$


which implies at once that for each $n,\left|f_{n}(2 \pi)\right|=1$ so that each $f_{n}(t)$ is the characteristic function of a lattice distribution with span equal to unity (cf. [3, pp. 59-60]).

LEMMA 2. Each distribution function corresponding to the factor $f_{n}(t)$ is bounded to the left.

Proof. Let $F_{n}(x)$ be the distribution function corresponding to $f_{n}(t)$. First we note that none of the distribution functions $F_{n}(x)$ can be a finite nondegenerate distribution, as in such a case (cf. [8, p. 141]) the corresponding characteristic function $f_{n}(z)$ is an entire function of the exponential type having infinitely many zeros. But clearly this is not possible, since none of the functions $f_{n}(z)$ can have any zeros in the entire complex plane. Therefore, each $F_{n}(x)$ must belong to one of the following three classes:

(A) $F_{n}(x)$ is unbounded on both sides so that it is purely discrete with jumps $p_{j n}$ $\left(p_{j n} \geqq 0, \sum_{j=-\infty}^{\infty} p_{j n}=1\right)$ at the points $\mu_{n}+j$ where $\mu_{n}$ is some real number and $j=0, \pm 1, \pm 2, \ldots$. Here without any loss of generality we can always choose $\mu_{n}$ so that $\sum_{j=0}^{\infty} p_{j n}<\frac{1}{2}$ and $\sum_{j=1}^{\infty} p_{-j n} \geqq \frac{1}{2}$. Therefore, we can write

$$
f_{n}(t)=\exp \left\{i t \mu_{n}\right\} \cdot \sum_{j=-\infty}^{\infty} p_{j n} \exp \{i t j\}=\exp \left\{i t \mu_{n}\right\} \phi_{n}(t),
$$

where

$$
\phi_{n}(t)=\sum_{j=-\infty}^{\infty} p_{j n} \exp \{i t j\}
$$

is again the characteristic function of a lattice distribution having jumps $p_{j n}$ at the points $j=0, \pm 1, \pm 2, \ldots$ so that $\sum_{j=1}^{\infty} p_{-j n} \geqq \frac{1}{2}$.

(B) $F_{n}(x)$ is bounded to the right so that it has jumps $p_{j n}$ only at the points $\mu_{n}-j$ where $\mu_{n}$ is some finite real number and $j=0,1,2, \ldots$ Here we can write

$$
f_{n}(t)=\exp \left\{i t \mu_{n}\right\} \sum_{j=0}^{\infty} p_{j n} \exp \{-i t j\}=\exp \left\{i t \mu_{n}\right\} \phi_{n}(t)
$$

where $\phi_{n}(t)=\sum_{j=0}^{\infty} p_{j n} \exp \{-i t j\}$ is the characteristic function of a lattice distribution which is bounded to the right by the point 0 having jumps $p_{j n}$ at the points $j=0,-1,-2, \ldots$

(C) $F_{n}(x)$ is bounded to the left so that it has jumps $p_{\text {fn }}$ only at the points $\mu_{n}+j$ where $\mu_{n}$ is some finite real number and $j=0,1,2, \ldots$ Here we write

$$
f_{n}(t)=\exp \left\{i t \mu_{n}\right\} \sum_{j=0}^{\infty} p_{j n} \exp \{i t j\}=\exp \left\{i t \mu_{n}\right\} \phi_{n}(t),
$$

where $\phi_{n}(t)=\sum_{j=0}^{\infty} p_{j n} \exp \{i t j\}$ is the characteristic function of a lattice distribution which is bounded to the left by the point 0 having jumps $p_{j n}$ at the points $j=$ $0,1,2, \ldots$ Hence the sequence $\left\{f_{n}(t)\right\}$ can be divided into three mutually exclusive and exhaustive subsequences $\left\{f_{n_{k}}(t)\right\},\left\{f_{n_{k}^{\prime}}^{\prime}(t)\right\}$ and $\left\{f_{n_{k}^{\prime}}(t)\right\}$ according as the corresponding distribution functions belong to the classes (A), (B) and (C) respectively. 
We write also the relations

$$
\begin{aligned}
& f_{n_{k}}(t)=\exp \left\{i t \mu_{n_{k}}\right\} \phi_{n_{k}}(t), \\
& f_{n_{k}}^{\prime}(t)=\exp \left\{i t \mu_{n_{k}^{\prime}}^{\prime}\right\} \phi_{n_{k}}^{\prime}(t), \\
& f_{n_{k}}^{\prime \prime}(t)=\exp \left\{i t \mu_{n_{k}^{\prime \prime}}^{\prime \prime}\right\} \phi_{n_{k}}^{\prime \prime}(t),
\end{aligned}
$$

corresponding to the classes (A), (B) and (C) respectively. We next substitute the relations (5a), (5b) and (5c) in (3) and thus obtain the relation

$$
\prod_{n=1}^{\infty} \exp \left\{i z \alpha_{n} \mu_{n}\right\}\left\{\phi_{n}(z)\right\}^{\alpha_{n}}=\exp \left\{\lambda\left(e^{i z}-1\right)\right\}
$$

holding for all $z$ in the complex plane where each factor $\phi_{n}(z)$ as a function of the complex variable $z$ is an entire characteristic function without zeros.

We put $z=2 \pi$ in (6) and note that $\phi_{n}(2 \pi)=1$ for every $n$ so that we have

$$
\prod_{n=1}^{\infty} \exp \left\{i 2 \pi \alpha_{n} \mu_{n}\right\}=\exp \left\{i 2 \pi \sum_{n=1}^{\infty} \alpha_{n} \mu_{n}\right\}=1
$$

and consequently $\sum_{n=1}^{\infty} \alpha_{n} \mu_{n}=m$ where $m$ is a certain finite integer which may be positive, negative or zero. Thus we obtain from (6)

$$
\prod_{n=1}^{\infty}\left\{\phi_{n}(z)\right\}^{\alpha_{n}}=\exp \left\{-i m z+\lambda\left(e^{i z}-1\right)\right\}
$$

for all complex $z$. We put $z=i v$ ( $(v$, real) in (7) and then get the relation

$$
\prod_{n=1}^{\infty}\left\{\dot{\phi}_{n}(i v)\right\}^{\alpha_{n}}=\exp \left\{m v+\lambda\left(e^{-v}-1\right)\right\}
$$

holding for all real $v$. We set (formally)

$$
\Phi_{1}(v)=\prod_{k}\left\{\phi_{n_{k}}(i v)\right\}^{\alpha_{n}}, \quad \Phi_{2}(v)=\prod_{k}\left\{\phi_{n_{k}^{\prime}}(i v)\right\}_{n_{k}^{\prime}}^{\alpha_{k}^{\prime}}, \quad \Phi_{3}(v)=\prod_{k}\left\{\phi_{n_{k}^{\prime \prime}}(i v)\right\}_{n_{k}^{\prime \prime}}^{\alpha_{n^{\prime}}} .
$$

First we verify easily from (4b) and (5b) that $\Phi_{2}(v)>1$ for $v>0$. Similarly we deduce from (4a) and (5a) that for $v>0$, each factor of $\Phi_{1}(v)$ has the estimate

$$
\phi_{n}(i v)>\sum_{j=1}^{\infty} p_{-j, n} \exp \{j v\}>\exp v \sum_{j=1}^{\infty} p_{-j n} \geqq \frac{1}{2} \exp v
$$

so that $\Phi_{1}(v)>1$ for $v>\ln 2$. We can also verify easily from (4c) and (5c) that $0 \leqq \Phi_{3}(v) \leqq 1$ for $v \geqq 0$. We now prove that $\Phi_{3}(v)$ cannot be identically zero for all $v>0$. We write $\psi(v)=\prod_{k} \phi_{n_{k}}^{\prime \prime}(i v)$ and see that $0 \leqq \psi(v) \leqq 1$ for $v \geqq 0$. Since $0<\alpha_{0}$ $\leqq \alpha_{n} \leqq 1$ and $0<\phi_{n_{k}^{\prime \prime}}^{\prime \prime}(i v) \leqq 1$ for $v \geqq 0$, we have for $v \geqq 0$

$$
0 \leqq \psi(v) \leqq \Phi_{3}(v) \leqq\{\psi(v)\}^{\alpha_{0}} \leqq 1 .
$$

First we show that $\Phi_{3} \not \equiv 0$. Let us suppose that $\Phi_{3}(v) \equiv 0$ for all $v>0$, then in such a case $\psi(v)$ is also identically zero. But $\psi(v)$ is a power-series in $e^{-v}$ with nonnegative 
coefficients and in this case all the coefficients must vanish and consequently $\psi(0)=0$. This contradicts the fact that $\psi(0)=\prod_{k} \phi_{n_{k}^{\prime \prime}}(0)=1$. Thus, for $v>0, \psi(v)$ cannot be identically zero so that it must contain at least one term which is a power of $e^{-v}$ with a certain positive coefficient. Therefore, for $v>0$, we have $\psi(v) \geqq A e^{-\gamma v}$ where $A>0$ and $\gamma$ a nonnegative integer. Thus for $v>0$ we get

$$
\Phi_{3}(v) \geqq \psi(v) \geqq A \exp \{-\gamma v\}
$$

We now turn to Lemma 2 and suppose that the statement of this lemma is not true. Then there exists at least one distribution function which is not bounded to the left but is either bounded or unbounded to the right. Therefore, there exists at least one characteristic function which belongs either to the set $\left\{\phi_{n_{k}}(t)\right\}$ or to the set $\left\{\phi_{n_{k}}^{\prime}(t)\right\}$. First we consider the case when it belongs to the set $\left\{\phi_{n_{k}}(t)\right\}$ and we denote this characteristic function by $\phi_{n_{1}}(t)$. We can then rewrite $\Phi_{1}(v)$ as

$$
\Phi_{1}(v)=\phi_{n_{1}}^{\alpha n_{1}}(i v) \prod_{k>1} \phi_{n_{k}}^{\alpha n_{k}}(i v)
$$

and verify easily that for $v>\ln 2, \Phi_{1}(v)>\phi_{n_{1}}^{\alpha_{n_{1}}}(i v) \geqq \phi_{n_{1}}^{\alpha}(i v)$. Thus for $v>\ln 2$ we obtain from (8) the estimate

$$
A \phi_{n_{1}^{0}}^{\alpha}(i v) \exp \{-(\gamma+m) v\}<\exp \{\lambda(\exp -v-1)\} .
$$

But $\phi_{n_{1}}(t)$ is the characteristic function of a distribution which is not bounded to the left and therefore we can always choose a sufficiently large positive integer $\nu$ subject to the condition $\nu \alpha_{0}-\gamma-m>0$ so that, for $\nu>0, \phi_{n_{1}}(i v)>B \exp \{v v\}$ where $0<B<1$. Thus we get finally for $v>\ln 2$

$$
A B^{\alpha_{0}} \exp \left\{\left(\nu \alpha_{0}-\gamma-m\right)\right\}<\exp \{\lambda(\exp -v-1)\} .
$$

But this relation cannot hold for sufficiently large values of $v$ since $\nu \alpha_{0}-\gamma-m>0$. Therefore there cannot exist any distribution function belonging to the class (A). Similarly we can show that there does not exist any distribution function belonging to the class (B). Therefore we conclude that each distribution function $F_{n}(x)$ must belong to the class $(\mathrm{C})$ thus completing the proof of Lemma 2.

Thus the functions $\phi_{n}(i v)$ satisfy the relation (8) where each $\phi_{n}(i v)$ has the form $\phi_{n}(i v)=\sum_{j=0}^{\infty} p_{j n} \exp \{-j v\}$ for all real $v$ according to $(4 \mathrm{c})$. We now prove that $m=\sum_{n=1}^{\infty} \alpha_{n} \mu_{n}=0$. For this purpose we introduce the sequence of characteristic functions $\psi_{N}(t)=\prod_{n=1}^{N} \phi_{n}(t)$ and denote the corresponding sequence of distribution functions by $H_{N}(x)(N=1,2, \ldots)$. We also write $\psi(t)=\prod_{n=1}^{\infty} \phi_{n}(t)$, which is the characteristic function of the limiting distribution function. We verify easily that for $v>0$

$$
0 \leqq \psi(i v) \leqq \prod_{n=1}^{\infty}\left\{\phi_{n}(i v)\right\}^{\alpha_{n}} \leqq\{\psi(i v)\}^{\alpha_{0}} \leqq 1
$$


so that, using the equation (8) and taking the logarithms, we obtain for $v>0$ the relation

$$
\ln \psi(i v) \leqq m v+\lambda(\exp -v-1) \leqq \alpha_{0} \ln \psi(i v) \leqq 0 .
$$

Thus for $v>0$, we have

$$
-\alpha_{0} \ln \psi(i v) / v \leqq-m+\dot{\lambda}, \quad(1-\exp -v) / v \leqq-(\ln \psi(i v)) / v .
$$

But the left extremity of each distribution function $H_{N}(x)$ is zero, so that, for any arbitrary $N$ and any $\varepsilon>0$, we have $H_{N}(-\varepsilon)=0$ while $H_{N}(\varepsilon)>0$. Therefore the left extremity of the limiting distribution function corresponding to the characteristic function $\psi(t)$ must also be zero. We now use Polya's formula (cf. [8, p. 140]) for the left extremity of a distribution and thus get

$$
-\limsup _{v \rightarrow \infty} \frac{\ln \psi(i v)}{v}=0 .
$$

We combine (11) and (12) and verify at once that $m=0$. We put $m=0$ in (7) and get the relation

$$
\prod_{n=1}^{\infty}\left\{\phi_{n}(z)\right\}^{\alpha_{n}}=\exp \{\lambda(\exp \{i z\}-1)\}
$$

holding for all complex $z$ where each factor $\phi_{n}(z)$ is an entire characteristic function without zeros and is of the form

$$
\phi_{n}(z)=\sum_{j=0}^{\infty} p_{j n} \exp \{i z j\}, \quad\left(p_{j n} \geqq 0, \sum_{j=0}^{\infty} p_{j n}=1\right) .
$$

Next we study the convergence of the infinite product $\prod_{n=1}^{\infty}\left\{\phi_{n}(z)\right\}^{\alpha_{n}}$.

LEMMA 3. The infinite product $\prod_{n=1}^{\infty}\left\{\phi_{n}(z)\right\}^{\alpha_{n}}$ converges uniformly in the circle $|z|<R(R$ is any arbitrary positive number).

Proof. First we note that for any $v$ in the interval $0 \leqq v \leqq R(R>0)$, we have, for any $n, 1 \leqq \phi_{n}(-i v) \leqq \phi_{n}(-i R)$ so that $0 \leqq \ln \phi_{n}(-i v) \leqq \ln \phi_{n}(-i R)$. We put $z=-i R$ $(R>0)$ in (13) and thus obtain

$$
\sum_{n=1}^{\infty} \alpha_{n} \ln \phi_{n}(-i R)=\lambda(\exp R-1) .
$$

Hence it follows from the convergence test of Weierstrass that the series

$$
\sum_{n=1}^{\infty} \alpha_{n} \ln \phi_{n}(-i v)
$$

converges uniformly in the interval $0 \leqq v \leqq R$. We construct the sequence of functions

$$
\theta_{N}(z)=\prod_{n=1}^{N} \phi_{n}(z), \quad N=1,2, \ldots,
$$


and consider the circle $|z|=R$. Since each $\phi_{n}(z)$ is regular and has no zeros inside the circle $|z|=R$, each function $\theta_{N}(z)$ is also regular and has no zeros inside this circle. Again from the form of $\phi_{n}(z)$, we can verify easily

$$
\max _{|z| \leqq R}\left|\phi_{n}(z)\right|=\phi_{n}(-i R) \geqq 1 \text {. }
$$

Therefore for every $N$ and every $z$ in $|z| \leqq R$, we have

$$
\left|\theta_{N}(z)\right|=\prod_{n=1}^{N}\left|\phi_{n}(z)\right|^{\alpha_{n}} \leqq \prod_{n=1}^{N}\left\{\phi_{n}(-i R)\right\}^{\alpha_{n}} \leqq \exp \{\lambda(\exp \{R\}-1)\} .
$$

Further the sequence of functions $\left\{\theta_{N}(z)\right\}$ converges uniformly on the segment of the imaginary axis $-R<v \leqq 0$ contained inside the circle $|z|=R$. Therefore according to the convergence theorem of Vitali (cf. [11, p. 168]) the infinite product

$$
\prod_{n=1}^{\infty}\left\{\phi_{n}(z)\right\}^{\alpha_{n}}
$$

converges uniformly in any circle $|z|<R$, so that Lemma 3 is proven.

Therefore the infinite product $\prod_{n=1}^{\infty}\left\{\phi_{n}(-i v)\right\}^{\alpha}$ converges uniformly in any finite $v$-interval $(|v|<R, R>0$ and arbitrary). We put $z=-i v$ ( $v$ real) in (13) and thus obtain the relation

$$
\prod_{n=1}^{\infty}\left\{\phi_{n}(-i v)\right\}^{\alpha_{n}}=\exp \{\lambda(\exp \{v\}-1)\}
$$

for all real $v$ where each $\phi_{n}(-i v)$ has the form

$$
\phi_{n}(-i v)=\sum_{j=0}^{\infty} p_{j n} \exp \{j v\} \quad\left(p_{j n} \geqq 0, \sum_{j=0}^{\infty} p_{j n}=1\right) .
$$

We take the limit of both sides of (14) as $v \rightarrow-\infty$ and thus obtain the relation

$$
\prod_{n=1}^{\infty} p_{0 n}^{\alpha_{n}}=\exp -\lambda
$$

so that each $p_{0 n}$ has the estimate $p_{n 0} \geqq \exp \left\{-\lambda / \alpha_{n}\right\} \geqq \exp \left\{-\lambda / \alpha_{0}\right\}>0$. We next use the transformation $x=\exp v(0 \leqq x<\infty)$ and introduce the sequence of functions

$$
g_{n}(x)=\sum_{j=0}^{\infty} p_{j n} x^{j} / p_{0 n}, \quad\left(p_{j n} \geqq 0, \sum_{j=0}^{\infty} p_{j n}=1\right)
$$

for $n=1,2, \ldots$ We note that each $g_{n}(x) \geqq 1$ for $x \geqq 0$ and further it is a power series in $x$ with nonnegative coefficients. Further we obtain the following lemma as an immediate consequence of Lemma 3 and the equation (14).

LEMMA 4. The infinite product $\prod_{n=1}^{\infty}\left\{g_{n}(x)\right\}^{\alpha_{n}}$ converges uniformly in the interval $0 \leqq x \leqq \Delta(\Delta>0$ and arbitrary) and further the relation

$$
\prod_{n=1}^{\infty}\left\{g_{n}(x)\right\}^{\alpha_{n}}=\exp \{\lambda x\}
$$

holds for all $x \geqq 0$. 
For further investigation we have to study the behavior of each function $g_{n}(w)$ as a complex valued function of the complex variable $w(w=x+i y, x$ and $y$ both real).

LEMMA 5. Each of the functions $g_{n}(w)$ ( $w$ complex) is regular and has no zeros at least in the circle $|w| \leqq \rho_{0}\left(\right.$ where $\left.\rho_{0}<\exp \left\{-\lambda / \alpha_{0}\right\}\right)$ about the point $w=0$.

Since

$$
g_{n}(w)=\sum_{j=0}^{\infty} p_{j n} w^{j} / p_{0 n}, \quad\left(p_{j n} \geqq 0, \sum_{j=0}^{\infty} p_{j n}=1\right),
$$

we have for every $n$ and every $w$ in $|w| \leqq 1$,

$$
\left|g_{n}(w)\right| \leqq \frac{1}{p_{0 n}} \sum_{j=0}^{\infty} p_{j n}|w|^{j} \leqq \frac{1}{p_{0 n}} \leqq \exp \left\{\lambda / \alpha_{0}\right\} \quad\left(\alpha_{0}>0\right)
$$

so that each $g_{n}(w)$ is regular at least in the circle $|w| \leqq 1$. Further for each $w$ in $|w| \leqq \rho_{0}\left(\right.$ where $\left.0<\rho_{0}<\exp \left\{-\lambda / \alpha_{0}\right\}\right)$ we have

so that

$$
\left|g_{n}(w)-1\right| \leqq \frac{1}{p_{0 n}} \sum_{j=1}^{\infty} p_{j n} \rho_{0}^{j} \leqq \frac{\rho_{0}}{p_{0 n}} \leqq \rho_{0} \exp \left\{\lambda / \alpha_{0}\right\}
$$

$$
\left|g_{n}(w)\right|>1-\rho_{0} \exp \left\{\lambda / \alpha_{0}\right\}>0
$$

and hence each $g_{n}(w)$ cannot have any zeros in this circle.

LEMMA 6. The relation

$$
\prod_{n=1}^{\infty}\left\{g_{n}(w)\right\}^{\alpha_{n}}=\exp \{\lambda w\}
$$

holds for all complex $w$ in any circle about the point $w=0$ in which each $g_{n}(w)$ is regular and has no zeros.

Proof. We consider a circle $|w|=R(R>0)$ about the point $w=0$ in which each $g_{n}(w)$ is regular and has no zeros. We construct a sequence of functions

$$
G_{N}(w)=\prod_{n=1}^{N}\left\{g_{n}(w)\right\}^{\alpha_{n}}, \quad N=1,2, \ldots
$$

and verify easily that each $G_{N}(w)$ is also regular and has no zeros inside the circle $|w|=R$. Each $g_{n}(w)$ is a power series in $W$ with nonnegative coefficients so that for each $n, \max _{|w| \leqq R}\left|g_{n}(w)\right|=g_{n}(R) \geqq 1$. We put $x=R$ in the equation (15) and get

$$
\prod_{n=1}^{\infty}\left\{g_{n}(R)\right\}^{\alpha_{n}}=\exp \{\lambda R\}
$$

Hence for every $N$ and every $w$ in $|w| \leqq R$ we have

$$
\left|G_{N}(w)\right|=\prod_{n=1}^{N}\left|g_{n}(w)\right|^{\alpha_{n}} \leqq \prod_{n=1}^{N}\left\{g_{n}(R)\right\}^{\alpha_{n}} \leqq \exp \{\lambda R\} .
$$


Further we note from Lemma 4 that the sequence $\left\{G_{N}(w)\right\}$ converges uniformly on the positive half segment of the real axis $0 \leqq x<R$ which is contained in the circle $|w|=R$. Therefore, according to the convergence theorem of Vitali, the sequence $\left\{G_{N}(w)\right\}$ converges uniformly inside the circle $|w|=R$ and further this limit function is also regular in the circle $|w|<R$. Then it follows immediately from the property of uniqueness of analytic continuation that equation (16) holds for all complex $w$ in the circle $|w|<R$.

LEMMA 7. Each function $g_{n}(w)$ has no zeros inside any circle about the point $w=0$ in which each $g_{n}(w)$ is regular.

Proof. Let $|w|=R_{0}\left(R_{0}>0\right)$ be a circle about the point $w=0$ in which each $g_{n}(w)$ is regular. Then clearly by virtue of Lemma 5 , we have $R_{0}>\rho_{0}>0$. We shall prove that each $g_{n}(w)$ cannot have any zeros inside the circle $|w|=R_{0}$. Let us suppose that the statement of this lemma is not true. Then there are zeros (finitely or possibly infinitely many) of the functions $g_{n}(w)$ inside this circle. Let $\rho_{1}$ be the infimum of the moduli of the set of all zeros of the functions $g_{n}(w)$ inside the circle $|w|=R_{0}$. Then clearly, according to Lemma 5, we have $\rho_{1}>\rho_{0}>0$. We suppose that $\rho_{1}<R_{0}$ so that $\delta=R_{0}-\rho_{1}>0$. Hence each of the functions $g_{n}(w)$ is regular and has no zeros in the circle $|w|<\rho_{1}$ and consequently according to Lemma 6 , equation (16) holds for all complex $w$ inside this circle. We set $X_{0}=\rho_{1}-\varepsilon / 2$ where $\varepsilon(0<\varepsilon<\delta)$ is a sufficiently small positive quantity and consider the circle $|w|=X_{0}$. We set $x=X_{0}+\xi$ and introduce the sequence of functions

$$
h_{n}(\xi)=g_{n}\left(X_{0}+\xi\right) / g_{n}\left(X_{0}\right), \quad n=1,2, \ldots
$$

It is easy to verify from Lemma 4 that the infinite product $\prod_{n=1}^{\infty}\left\{h_{n}(\xi)\right\}^{\alpha_{n}}$ converges uniformly in any interval $0 \leqq \xi \leqq \xi_{0}\left(\xi_{0}>0\right.$ and arbitrary) and that the relation

$$
\prod_{n=1}^{\infty}\left\{h_{n}(\xi)\right\}^{\alpha_{n}}=\exp \{\lambda \xi\}
$$

holds for all $\xi \geqq 0$. Each $h_{n}(\xi) \geqq 1$ for $\xi \geqq 0$ and further it is a power series in $\xi$ with nonnegative coefficients. Therefore we can write

$$
h_{n}(\xi)=\frac{g_{n}\left(X_{0}+\xi\right)}{g_{n}\left(X_{0}\right)}=\sum_{j=0}^{\infty} q_{j n} \xi^{j}
$$

where $q_{0 n}=h_{n}(0)=1$ and $q_{j n} \geqq 0(j=1,2, \ldots ; n=1,2, \ldots)$. Using (17) we obtain for every $n$, the estimate

$$
\sum_{j=0}^{n} q_{j n}=h_{n}(1) \leqq \exp \left\{\lambda / \alpha_{n}\right\} \leqq \exp \left\{\lambda / \alpha_{0}\right\} .
$$

We now consider each $h_{n}(\zeta)$ as a function of the complex variable $\zeta$ and see easily that for every $n$ and every $\zeta$ in $|\zeta| \leqq 1$

$$
\left|h_{n}(\zeta)\right| \leqq \sum_{j=0}^{\infty} q_{j n}|\zeta|^{j} \leqq \sum_{j=0}^{\infty} q_{j n} \leqq \exp \left\{\lambda / \alpha_{0}\right\}
$$


so that each $h_{n}(\zeta)$ is regular at least in the circle $|\zeta|<1$ about the point $\zeta=0$. Further for every $n$ and every $\zeta$ in $|\zeta| \leqq \varepsilon<1$ we have the estimate

$$
\left|h_{n}(\zeta)-1\right| \leqq \sum_{j=1}^{n} q_{j n} \varepsilon^{j} \leqq \varepsilon \sum_{j=0}^{n} q_{j n} \leqq \varepsilon \exp \left\{\lambda / \alpha_{0}\right\}
$$

so that for every $n$ and every $\varepsilon$ in $0<\varepsilon<1$, we have $h_{n}(\varepsilon)-1 \leqq \varepsilon \exp \left\{\lambda / \alpha_{0}\right\}$ so that

$$
g_{n}\left(X_{0}+\varepsilon\right)-g_{n}\left(X_{0}\right) \leqq \varepsilon \exp \left\{\lambda / \alpha_{0}\right\} g_{n}\left(X_{0}\right) .
$$

Again putting $x=X_{0}$ in (15) we obtain $g_{n}\left(X_{0}\right) \leqq \exp \left\{\lambda X_{0} / \alpha_{0}\right\}<\exp \left\{\lambda \rho_{1} / \alpha_{0}\right\}$ so that we obtain finally for every $n$ and every $\varepsilon$ in $0<\varepsilon<1$ and $X_{0}>0$, the estimate

$$
g_{n}\left(X_{0}+\varepsilon\right)-g_{n}\left(X_{0}\right)<\varepsilon \exp \left\{\lambda\left(1+\rho_{1}\right) / \alpha_{0}\right\} .
$$

Let $W_{0}$ be an arbitrary point on the circumference of the circle $|w|=X_{0}$. Then clearly according to our assumption each $g_{n}\left(W_{0}+\zeta\right)$ as a function of the complex variable $\zeta$ is regular at least in the circle $|\zeta|<\varepsilon$ and admits the McLaurin expansion so that for every $n$ and every $\zeta$ in $|\zeta|<\varepsilon$, we have

$$
\left|g_{n}\left(W_{0}+\zeta\right)-g_{n}\left(W_{0}\right)\right| \leqq \sum_{k=1}^{\infty} \frac{\varepsilon^{k}}{k !}\left|g_{n}^{(k)}\left(W_{0}\right)\right|
$$

But for any $k$ and $n$ the function $g_{n}^{(k)}\left(W_{0}\right)$ is again a power series in $W_{0}$ with nonnegative coefficients so that for any $W_{0}$ on the circle $|w|=X_{0}$ we have $\left|g_{n}^{(k)}\left(W_{0}\right)\right|$ $\leqq g_{n}^{(k)}\left(X_{0}\right)$. Hence we have

$$
\left|g_{n}\left(W_{0}+\zeta\right)-g_{n}\left(W_{0}\right)\right| \leqq \sum_{k=1}^{\infty} \frac{\varepsilon^{k}}{k !} g_{n}^{(k)}\left(X_{0}\right)=g_{n}\left(X_{0}+\varepsilon\right)-g_{n}\left(X_{0}\right)
$$

Then using (18) we get

$$
\left|g_{n}\left(W_{0}+\zeta\right)-g_{n}\left(W_{0}\right)\right|<\varepsilon \exp \left\{\lambda\left(1+\rho_{1}\right) / \alpha_{0}\right\} .
$$

Next we proceed to obtain an estimate for $\left|g_{n}\left(W_{0}\right)\right|$. Clearly equation (16) holds for all complex $W$ on the circumference of the circle $|w|=X_{0}$ so that we have

$$
\prod_{n=1}^{\infty}\left|g_{n}\left(W_{0}\right)\right|^{\alpha_{n}}=\left|\exp \left\{\lambda W_{0}\right\}\right| \geqq \exp \left\{-\lambda X_{0}\right\}
$$

and

$$
\prod_{n=1}^{\infty}\left\{g_{n}\left(X_{0}\right)\right\}^{\alpha_{n}}=\exp \left\{\lambda X_{0}\right\}
$$

so that

$$
\prod_{n=1}^{\infty}\left\{\frac{\left|g_{n}\left(W_{0}\right)\right|}{g_{n}\left(X_{0}\right)}\right\}^{\alpha_{n}} \geqq \exp \left\{-2 \lambda X_{0}\right\} .
$$

Again we note that for every $n,\left|g_{n}\left(W_{0}\right)\right| \leqq g_{n}\left(X_{0}\right)$ so that we have the estimate

$$
\left|g_{n}\left(W_{0}\right)\right| \geqq g_{n}\left(X_{0}\right) \exp \left\{-2 \lambda X_{0} / \alpha_{0}\right\} \geqq \exp \left\{-2 \lambda X_{0} / \alpha_{0}\right\}>\exp \left\{-2 \lambda \rho_{1} / \alpha_{0}\right\} .
$$


We now combine (19) and (20) and thus obtain for every $n$ and every $W_{0}$ on the circle $|w|=X_{0}$ and every $\zeta$ in $|\zeta|<\varepsilon$, the estimate

$$
\left|g_{n}\left(W_{0}+\zeta\right)\right|>\exp \left\{-2 \lambda \rho_{1} / \alpha_{0}\right\}-\varepsilon \exp \left\{\lambda\left(1+\rho_{1}\right) / \alpha_{0}\right\} .
$$

Therefore for $\varepsilon<\min \left(\delta, \exp \left\{-\lambda\left(1+3 \rho_{1}\right) / \alpha_{0}\right\}\right)$ we obtain $\left|g_{n}\left(W_{0}+\zeta\right)\right|>0$. Thus we conclude that there always exists a circle of radius $\varepsilon$ around every point $W_{0}$ on the circumference of the circle $|w|=X_{0}=\rho_{1}-\varepsilon / 2$ in which none of the functions $g_{n}(w)$ has any zeros so that each $g_{n}(w)$ has no zeros at least inside the circle $|w|<\rho_{1}+\varepsilon / 2$. But this contradicts our assumption that $\rho_{1}$ is the infimum of the set of all zeros of the functions $g_{n}(w)$. Hence $\rho_{1} \geqq R_{0}$ and each $g_{n}(w)$ cannot have any zeros inside the circle $|w|=R_{0}$.

LEMMA 8. Each of the functions $g_{n}(w)$ is an entire function.

Proof. Let us suppose that the statement of the lemma is not true. Then there exists at least one $g_{n}(w)$ which converges only in a circle about the point $w=0$ with a certain finite radius of convergence. Let $\rho_{2}$ be the infimum of the radii of convergence of the functions $g_{n}(w)$. Then clearly according to Lemma 5, we have $\rho_{2}>\rho_{0}>0$. Then according to our assumption each of the functions $g_{n}(w)$ is regular in the circle $|w|<\rho_{2}$ about the point $w=0$. Consequently according to Lemma 7 each $g_{n}(w)$ has no zeros inside this circle and further by virtue of Lemma 6, equation (16) holds for all complex $w$ in this circle. We now set $X_{0}=\rho_{2}-\varepsilon$ where $\varepsilon$ is a sufficiently small positive quantity. We substitute $x=X_{0}+\xi$ ( $\xi$ real) and introduce the functions

$$
h_{n}(\xi)=g_{n}\left(X_{0}+\xi\right) / g_{n}\left(X_{0}\right), \quad n=1,2, \ldots
$$

We then proceed in the same manner as in the proof of Lemma 7 and prove that each $h_{n}(\zeta)$ as a function of the complex variable $\zeta$ converges in a circle about the point $\zeta=0$ with a radius of convergence which is not less than unity. Each $g_{n}(w)$ is a power series with nonnegative coefficients and hence it follows from the theorem of Pringsheim and Landau that the point of singularity of the function $g_{n}(w)$ which is nearest to the origin $w=0$ must be located on the positive half segment of the real axis. Thus we deduce that each $g_{n}(w)$ is regular at least in the circle $|w|<\rho_{2}+(1-\varepsilon)$. But this contradicts our assumption that $\rho_{2}$ is the infimum of the radii of convergence of the functions $g_{n}(w)$. Therefore each $g_{n}(w)$ is an entire function.

LEMMA 9. Each of the functions $g_{n}(w)$ has the form

$$
g_{n}(w)=\exp \left\{\lambda_{n} w\right\}, \quad \lambda_{n} \geqq 0 \quad(n=1,2, \ldots) .
$$

Proof. As a direct consequence of Lemmas 6, 7, and 8, we conclude that each $g_{n}(w)$ is an entire function and has no zeros in the whole complex plane and further equation (16) holds for all complex $w$ in the plane. We set $w=R$ in (16) and obtain for every $n$ the estimate

$$
\max _{|w|=R}\left|g_{n}(w)\right|=g_{n}(R) \leqq \exp \left\{\lambda R / \alpha_{0}\right\} \quad\left(\alpha_{0}>0\right)
$$


so that

$$
\max _{|w|=R}\left|g_{n}(w)\right|=O(\exp \{a R\}) \quad \text { as } R \rightarrow \infty \quad(a>0) .
$$

Therefore the order of each entire function $g_{n}(w)$ cannot exceed unity and it follows at once from the factorization theorem of Hadamard that each $g_{n}(w)$ has the form

$$
g_{n}(w)=\exp \left(\lambda_{n} w+\nu_{n}\right), \quad n=1,2, \ldots
$$

But we further note that each $g_{n}(0)=1$ so that $\nu_{n}=0$ and each $g_{n}(x) \geqq 1$ for $x>0$ so that $\lambda_{n}$ is real and nonnegative. This completes the proof of Lemma 9.

We now turn to the proof of the Theorem. It is very easy to verify from Lemma 9 that each factor $\phi_{n}(t)$ has the form

$$
\phi_{n}(t)=\exp \left\{\lambda_{n}(\exp \{i t\}-1)\right\}, \quad n=1,2, \ldots
$$

We now note that

$$
f_{n}(t)=\exp \left\{i \mu_{n} t\right\} \phi_{n}(t), \quad n=1,2, \ldots,
$$

and thus complete the proof of the theorem.

\section{REFERENCES}

1. H. Cramér, Über eine Eigenschaft der normalen Verteilungsfunktion, Math. Z. 41 (1936), 405-414.

2. D. Dugué, Sur le théorème de Lévy-Cramér, Publ. Inst. Statist. Univ. Paris 6 (1957), 213-225.

3. B. V. Gnedenko and A. N. Kolmogorov, Limit distributions for sums of independent random variables, English transl., K. L. Chung, Addison-Wesley, Reading, Mass., 1954.

4. R. G. Laha, On a factorization theorem in the theory of analytic characteristic functions, Ann. Math. Statist. 28 (1957), 126-139.

5. - On functions having non-negative derivatives at the origin, Proc. Amer. Math. Soc. 12 (1961), 175-180.

6. Yu. V. Linnik, On “ $\alpha$-decomposition" of infinitely divisible laws, Vestnik Leningrad. Univ. Ser. Mat. Meh. Astronom. 14 (1959), 14-23. (Russian)

7. Yu. V. Linnik and A. A. Zinger, On an analytical extension of a theorem of Cramér and its application, Vestnik Leningrad. Univ. Ser. Mat. Meh. Astronom. 10 (1955), 51-56. (Russian)

8. E. Lukacs, Characteristic functions, Griffin, London, 1960.

9. L. V. Mamay, On the theory of characteristic functions, Vestnik Leningrad. Univ. Ser. Mat. Meh. Astronom. 15 (1960), 85-90. (Russian)

10. D. A. Raikov, On the decomposition of Gauss and Poisson laws, Izv. Akad. Nauk SSSR Ser. Mat. 2 (1938), 91-124. (Russian)

11. E. C. Titchmarsh, The theory of functions, Oxford Univ. Press, New York, 1939.

Catholic University of America, WASHINGTON, D.C. 